Vietnam Journal of Mechanics, VAST, Vol.33, No. 1 (2011), pp. $27-40$

\title{
BENDING VIBRATION OF BEAM ELEMENTS UNDER MOVING LOADS WITH CONSIDERING VEHICLE BRAKING FORCES
}

\author{
Nguyen Xuan Toan \\ Da Nang University of Technology
}

\begin{abstract}
The study of fluctuations of structures in general and bridge structures in particular under the influence of moving loads considering the impact of vehicle braking forces draws the attention of many scientists. However, due to the complexity of this problem a static method has been so far applied for approximate calculation in bridge design standards. In this article the author introduces the equation of bending vibrations of beam elements according to the model of dynamic interaction between beam elements and moving vehicle loads considering vehicle braking forces.

Key words: bending vibration, braking force, moving load.
\end{abstract}

\section{INTRODUCTION}

The impact of vehicle braking forces on the bridge is huge and must be considered in design. In the bridge design process of many countries it is imperative to audit vehicle braking force bearing structures. Due to the complexity of this problem in the current processes only vehicle braking force bearing structures have been audited in accordance with a static method based on standard conventional loads. However, the neglecting of dynamic effects of vehicle - bridge interaction may result in large errors $[1,2]$. Today modern bridges tend to use high - strength materials, their structure is very slender and their hardness is small; therefore, they are very sensitive to cyclic impact loads, especially, large ones of vehicles moving at high-speeds. As a result, the study of the vibrations of bridge structures enduring/bearing moving loads has been interested by many scientists $[3]-[13],[15]-[18]$.

In reality, the fact that vehicles brake on bridges causes very large vibrations, so the study of bridge structure vibrations enduring moving loads considering the impact of vehicle braking forces is of great importance and urgency. In this paper, the author introduces a model of dynamic interaction between beam elements and moving vehicle loads, namely, a three-mass model considering vehicle braking forces. A corresponding system of differential equations of bending vibrations of the beam element considering vehicle braking forces is obtained. 


\section{COMPUTATIONAL MODEL AND ASSUMPTIONS}

The three - mass model of dynamic interaction between the beam elements and moving vehicle loads, considering vehicle braking forces and the coordinate axes on elements are described as in Fig. 1 and Fig. 2.

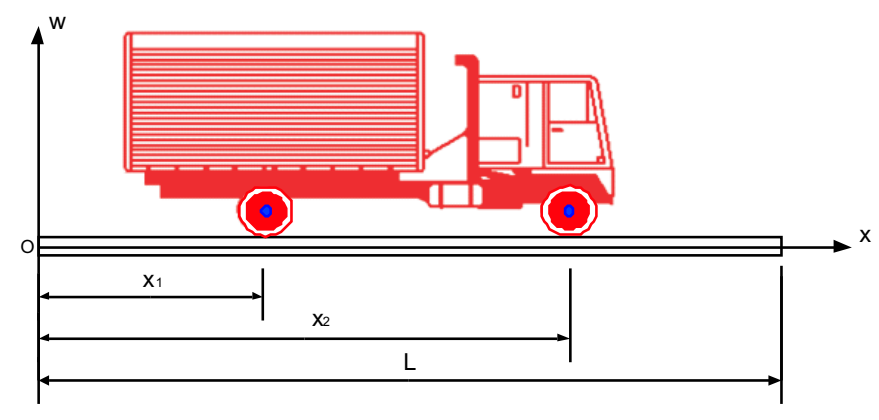

Fig. 1. Diagram of 2- axes vehicles on the beam element

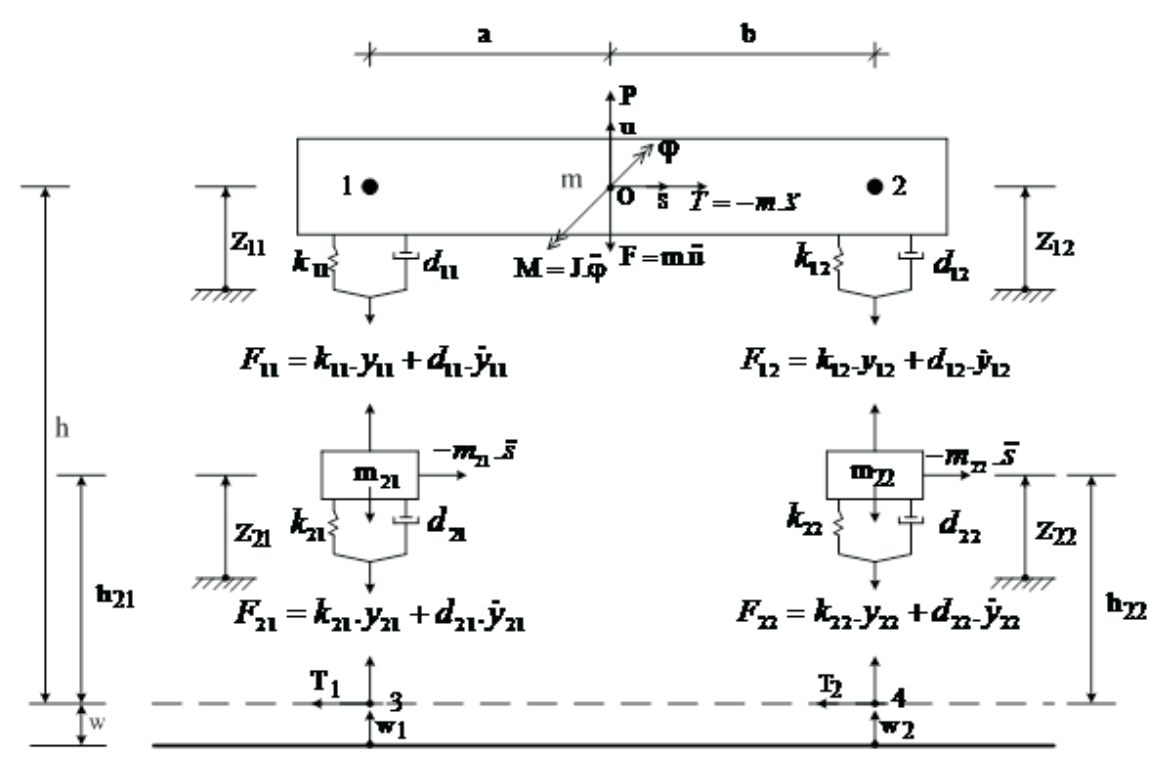

Fig. 2. Interaction model between two axes and beam elements

One has

$$
x_{i}= \begin{cases}v_{i} \cdot\left(t-t_{i}\right), & \text { when } t_{i} \leq t \leq t_{h i} . \\ v_{i} \cdot\left(t_{h i}-t_{i}\right)+\left[\frac{a_{i} \cdot\left(t-t_{h i}\right)}{2}+v_{i}\right] \cdot\left(t-t_{h i}\right), & \text { when } t_{h i}<t \leq t_{e i} .\end{cases}
$$

It is denoted (see Fig. 1 and Fig. 2): $L$ - the length of the beam elements being considered 
$x_{i}$ - the $\mathrm{i}^{\text {th }}$ vehicle coordinate axes at the time being considered

$v_{i}$ - the velocity of the $\mathrm{i}^{\text {th }}$ axle before braking

$a_{i}$ - the acceleration of the $\mathrm{i}^{\text {th }}$ axle when braking

$t_{i}$ - the time when the $\mathrm{i}^{\text {th }}$ axle begins running on the beam elements

$t_{h i}$ - the time when the $\mathrm{i}^{\text {th }}$ axle begins braking

$t_{e i}$ - the time when the $\mathrm{i}^{\text {th }}$ axle was at the end of the element

$t$ - the time being considered

$P=G \cdot \sin (\Omega . t+\alpha)$ the conditioning stimulation force caused by the eccentric mass of the engine

$m$ - the mass of the entire vehicle and goods, excluding the mass of the axle

$m_{21}$ - the mass of the $1^{s t}$ axle

$m_{22}$ - the mass of the $2^{\text {nd }}$ axle

$k_{11}, d_{11}$ - hardness and damping rate of the $1^{\text {st }}$ cart spring

$k_{21}, d_{21}$ - hardness and damping rate of the $1^{\text {st }}$ tire

$k_{12}, d_{12}$ - hardness and damping rate of cart spring $2^{n d}$

$k_{22}, d_{22}$ - hardness and damping rate of the $2^{\text {nd }}$ tire

$z_{11}$ - absolute displacement of the chassis at the $1^{\text {st }}$ axle

$z_{21^{-}}$absolute displacement of the $1^{\text {st }}$ axle, absolute coordinates of the mass $m_{21}$

$z_{12^{-}}$absolute displacement of the chassis at the $2^{\text {st }}$ axle

$z_{22}$ absolute displacement of the $2^{t}$ axle, absolute coordinates of the mass $m_{22}$

$y_{11}$ - relative displacement between the chassis and the $1^{\text {st }}$ axle

$y_{21}$ - relative displacement between the beam element and the $1^{\text {st }}$ axle

$y_{12}$ - relative displacement between the chassis and the $2^{s t}$ axle

$y_{22}$ - relative displacement between the beam element and the $2^{\text {st }}$ axle

$u$ - absolute displacement of the chassis at heart block (absolute coordinate of the mass $m$ )

$\varphi$ - the rotation angle of the vehicle tank

$s$ - the stretch of road that vehicles move on

$a, b$ - the distance from the center of mass $\mathrm{O}$ to the $1^{\text {st }}$ and the $2^{s t}$ axles

$T_{1}, T_{2}$ - the friction forces between tyre and bridge surface when braking

Inertial forces, dray forces, elastic forces, exciting forces and braking forces affecting the system as shown in Fig. 2 have conventional dimensions and sign in accordance with the system of corresponding coordinate axes.

The following assumptions are adopted:

The mass of the entire vehicle and goods, excluding the mass of the axle is transferred to the center of mass system. It is equivalent to the mass $\mathrm{m}$ and the rotational inertia $\mathrm{J}$.

The mass of the $1^{s t}$ and $2^{\text {nd }}$ axles is $m_{21}$ and $m_{22}$, which are regarded as a point with concentrated mass at the center of the corresponding axle.

The chassis is hypothesised to be absolutely hard and undistorted when moving.

The vertical displacements of mass $m, m_{21}, m_{22}$ are smaller than the height from their center to the centre of beam.

Beam materials work in the linear elastic stage. 
The bridge surface is flat, and has the friction coefficient homogeneous over the entire bridge surface.

Brake forces of axles of vehicle are assumed to occur simultaneously. The direction of the forces between bridge surface and tires are assumed to be in the opposite direction of movement of vehicle as shown in Fig. 2.

According to this assumption, the brake forces between bridge surface and tires, called $T_{1}, T_{2}$, make the vehicle decelerates uniformly and cause inertia forces $-m_{21} . \ddot{s}$, $-m_{22} . \ddot{s},-m . \ddot{s}$. These inertia forces which in turn produce longitudinal and vertical oscillations of the whole system.

The most dangerous case is when an emergency brake is applied. In this case, the forces $T_{1}, T_{2}$ are assumed to be directly proportional to loaded weight of vehicle:

$$
T_{1}+T_{2}=\left(m+m_{21}+m_{22}\right) \cdot g \cdot \tau
$$

$\tau$ - the friction factor between bridge surface and tires

$g$ - the acceleration of gravity.

\section{DIFFENTIAL EQUATIONS OF MOVING LOADS}

Based on the calculation model and assumptions in Section 1, we consider the system of mass $m, m_{21}, m_{22}$, viscous drag, elastic forces, inertial forces, stimulation forces, bridge surface constraint forces, braking power, which are converted to frictional forces against the bridge surface as shown in Fig. 2.

Applying the principle of d'Alembert, considering the balance of each mass $m, m_{21}$, $m_{22}$ according to the vertical axis and the whole system according to the horizontal axis, we have:

$$
\begin{aligned}
& P-m \ddot{u}-F_{11}-F_{12}-m g=0 \\
& F_{11}-F_{21}-m_{21} \ddot{z}_{21}-m_{21} g=0 \\
& F_{12}-F_{22}-m_{22} \ddot{z}_{22}-m_{22} g=0 \\
& T_{1}+T_{2}=-\left(m+m_{21}+m_{22}\right) \ddot{s}
\end{aligned}
$$

Similarly, considering the torque balance of the whole system with the $3^{\text {rd }}$ points:

$(m \ddot{u}+m g-P) \cdot a-\left(m \cdot h+m_{21} \cdot h_{21}+m_{22} \cdot h_{22}\right) \ddot{s}-J \ddot{\varphi}+\left(m_{22} \cdot \ddot{z}_{22}+m_{22} \cdot g+F_{22}\right) \cdot(a+b)=0$

in which:

$$
\begin{aligned}
& F_{11}=k_{11} \cdot y_{11}+d_{11} \cdot \dot{y}_{11}, F_{12}=k_{12} \cdot y_{12}+d_{12} \cdot \dot{y}_{12} \\
& F_{21}=k_{21} \cdot y_{21}+d_{21} \cdot \dot{y}_{21}, F_{22}=k_{22} \cdot y_{22}+d_{22} \cdot \dot{y}_{22} \\
& \varphi=\left(z_{11}-z_{12}\right) /(a+b), u=\left(b \cdot z_{11}+a \cdot z_{12}\right) /(a+b) \\
& z_{11}=y_{11}+y_{21}+w_{1}, z_{12}=y_{12}+y_{22}+w_{2} \\
& z_{21}=y_{21}+w_{1}, z_{22}=y_{22}+w_{2}
\end{aligned}
$$

Combining (2) with (3), (4) and (5) then having them transformed, we obtain a set of equations: 


$$
\begin{array}{r}
m J \ddot{z}_{11}+\left(a^{2} m+J\right) d_{11} \dot{z}_{11}-(m b a-J) d_{12} \dot{z}_{12}-\left(a^{2} m+J\right) d_{11} \dot{z}_{21}+(m b a-J) d_{12} \dot{z}_{22}+ \\
\left(a^{2} m+J\right) k_{11} z_{11}-(m b a-J) k_{12} z_{12}-\left(a^{2} m+J\right) k_{11} z_{21}+ \\
+(m b a-J) k_{12} z_{22}-J P+J \mathrm{Jg}+\left(\mathrm{m} \cdot \mathrm{h}+\mathrm{m}_{21} \cdot h_{21}+m_{22} \cdot h_{22}\right) \cdot m a \cdot \ddot{s}=0 \\
m J \ddot{z}_{12}+(m b a+J) d_{11} \dot{z}_{11}+\left(b^{2} m+J\right) d_{12} \dot{z}_{12}-(m b a+J) d_{11} \dot{z}_{21}-\left(b^{2} m+J\right) d_{12} \dot{z}_{22}+ \\
+(m b a+J) k_{11} z_{11}+\left(b^{2} m+J\right) k_{12} z_{12}-(m b a+J) k_{11} z_{21}- \\
-\left(b^{2} m+J\right) k_{12} z_{22}-J P+\mathrm{Jmg}+\left(\mathrm{m} \cdot \mathrm{h}+\mathrm{m}_{21} \cdot h_{21}+m_{22} \cdot h_{22}\right) \cdot m b \cdot \ddot{s}=0 \\
m_{21} \ddot{z}_{21}-d_{11} \dot{z}_{11}+\left(d_{11}+d_{21}\right) \dot{z}_{21}-k_{11} z_{11}+\left(k_{11}+k_{21}\right) z_{21}+\mathrm{m}_{21} \cdot g-d_{21} \cdot \dot{w}_{1}-k_{21} \cdot w_{1}=0 \\
m_{22} \ddot{z}_{22}-d_{12} \dot{z}_{12}+\left(d_{12}+d_{22}\right) \dot{z}_{22}-k_{12} z_{12}+\left(k_{12}+k_{22}\right) z_{22}+\mathrm{m}_{22} \cdot g-d_{22} \cdot \dot{w}_{2}-k_{22} \cdot w_{2}=0 \\
\ddot{s}=-g \cdot \tau
\end{array}
$$

The constraint forces $F_{21}$ and $F_{22}$ are as follows:

$$
\begin{aligned}
& F_{21}=-m_{21} \ddot{z}_{21}+\left(\dot{z}_{11}-\dot{z}_{21}\right) d_{11}+\left(z_{11}-z_{21}\right) k_{11} \\
& F_{22}=-m_{22} \ddot{z}_{22}+\left(\dot{z}_{12}-\dot{z}_{22}\right) d_{12}+\left(z_{12}-z_{22}\right) k_{12}
\end{aligned}
$$

Having them rewritten in the form of distribution and adding a logic control signal function, we have:

$$
\begin{aligned}
& p_{1}(x, z, t)=\xi_{1}(t) \cdot\left[-m_{21} \ddot{z}_{21}+\left(\dot{z}_{11}-\dot{z}_{21}\right) d_{11}+\left(z_{11}-z_{21}\right) k_{11}\right] \delta\left(x-x_{1}\right) \\
& p_{2}(x, z, t)=\xi_{2}(t) \cdot\left[-m_{22} \ddot{z}_{22}+\left(\dot{z}_{12}-\dot{z}_{22}\right) d_{12}+\left(z_{12}-z_{22}\right) k_{12}\right] \delta\left(x-x_{2}\right)
\end{aligned}
$$

in which: $\xi_{i}(t)=\left\{\begin{array}{ll}1 & \text { when } t_{i} \leq t \leq t_{i}+\Delta T_{i} \\ 0 & \text { when } t<t_{i} \text { and } t>t_{i}+\Delta T_{i}\end{array}\right.$ is a logic control signal function, $\delta\left(x-x_{i}\right)$ is the Dirac delta function,

$\Delta T_{i}$ is the period of time that the $\mathrm{i}^{\text {th }}$ axle runs on the beam elements being considered.

\section{EQUATIONS OF BENDING VIBRATION OF BEAM ELEMENTS UNDER MOVING LOADS}

According to [16] the equation of bending vibrations of beam elements under distributed load $p(x, z, t)$ considering the effects of internal and external friction can be written as follows:

$$
E J_{d} \cdot\left(\frac{\partial^{4} w}{\partial x^{4}}+\theta \cdot \frac{\partial^{5} w}{\partial x^{4} \cdot \partial t}\right)+\rho F_{d} \cdot \frac{\partial^{2} w}{\partial t^{2}}+\beta \cdot \frac{\partial w}{\partial t}=p(x, z, t)=p_{1}(x, z, t)+p_{2}(x, z, t)
$$

in which $p_{1}(x, z, t)$ and $p_{2}(x, z, t)$ are determined by the formula $(7)$,

$E J_{d}$ - the bending stiffness of beam elements,

$\rho F_{d}$ - the mass of the beam element on a length unit,

$\theta$ and $\beta$ - the coefficient of internal friction and coefficient of external friction, respectively.

Aggregating (6) with (8) we have systems of differential equations of bending vibrations of beam elements under the influence of moving loads taking into account the 
impact of vehicle braking forces:

$$
\begin{array}{r}
E J_{d} \cdot\left(\frac{\partial^{4} w}{\partial x^{4}}+\theta \cdot \frac{\partial^{5} w}{\partial x^{4} \cdot \partial t}\right)+\rho F_{d} \cdot \frac{\partial^{2} w}{\partial t^{2}}+\beta \cdot \frac{\partial w}{\partial t}=p(x, z, t)=p_{1}(x, z, t)+p_{2}(x, z, t) m J \ddot{z}_{11}+ \\
+\left(a^{2} m+J\right) d_{11} \dot{z}_{11}-(m b a-J) d_{12} \dot{z}_{12}-\left(a^{2} m+J\right) d_{11} \dot{z}_{21}+(m b a-J) d_{12} \dot{z}_{22}+ \\
+\left(a^{2} m+J\right) k_{11} z_{11}-(m b a-J) k_{12} z_{12}-\left(a^{2} m+J\right) k_{11} z_{21}+(m b a-J) k_{12} z_{22}- \\
-\mathrm{JP}+\mathrm{Jmg}+\left(\mathrm{m} \cdot \mathrm{h}+\mathrm{m}_{21} \cdot h_{21}+m_{22} \cdot h_{22}\right) \cdot m a . \ddot{s}=0 \\
m J \ddot{z}_{12}+(m b a+J) d_{11} \dot{z}_{11}+\left(b^{2} m+J\right) d_{12} \dot{z}_{12}-(m b a+J) d_{11} \dot{z}_{21}-\left(b^{2} m+J\right) d_{12} \dot{z}_{22}+ \\
+(m b a+J) k_{11} z_{11}+\left(b^{2} m+J\right) k_{12} z_{12}-(m b a+J) k_{11} z_{21}-\left(b^{2} m+J\right) k_{12} z_{22}- \\
-\mathrm{JP}+\mathrm{Jmg}+\left(\mathrm{m} \cdot \mathrm{h}+\mathrm{m}_{21} \cdot h_{21}+m_{22} \cdot h_{22}\right) \cdot m b \cdot \ddot{s}=0 \\
m_{21} \ddot{z}_{21}-d_{11} \dot{z}_{11}+\left(d_{11}+d_{21}\right) \dot{z}_{21}-k_{11} z_{11}+\left(k_{11}+k_{21}\right) z_{21}+\mathrm{m}_{21} \cdot g-d_{21} \cdot \dot{w}_{1}-k_{21} \cdot w_{1}=0 \\
m_{22} \ddot{z}_{22}-d_{12} \dot{z}_{12}+\left(d_{12}+d_{22}\right) \dot{z}_{22}-k_{12} z_{12}+\left(k_{12}+k_{22}\right) z_{22}+\mathrm{m}_{22} \cdot g-d_{22} \cdot \dot{w}_{2}-k_{22} \cdot w_{2}=0 \\
\ddot{s}=-g \cdot \tau
\end{array}
$$

\section{TRANSFORMATION OF THE EQUATION OF BENDING VIBRATIONS OF BEAM ELEMENT TO THE MATRIX FORM}

The bending vibration we can be approximately presented in the form [16, 19]:

$$
\begin{gathered}
w=\left[\begin{array}{llll}
N_{1} & N_{2} & N_{3} & N_{4}
\end{array}\right] \cdot\left\{\begin{array}{c}
w_{1} \\
\varphi_{1} \\
w_{2} \\
\varphi_{2}
\end{array}\right\} \\
N_{1}=\frac{1}{L^{3}}\left(L^{3}-3 L x^{2}+2 x^{3}\right), N_{2}=\frac{1}{L^{2}}\left(L^{2} x-2 L x^{2}+x^{3}\right) \\
N_{3}=\frac{1}{L^{3}}\left(3 L x^{2}-2 x^{3}\right), N_{4}=\frac{1}{L^{2}}\left(x^{3}-L x^{2}\right)
\end{gathered}
$$

where: $w_{1}, \varphi_{1}$ - the deflection and rotation angle of the left end of beam element,

$w_{2}, \varphi_{2}$ - the deflection and rotation angle of the right end of beam element.

Substituting (10), (11) into (9) and applying the Galerkin method in combination with Green theory, we integrate each term by parts and obtain:

$$
\begin{aligned}
& \int_{0}^{L}\left\{\begin{array}{c}
N_{1} \\
N_{2} \\
N_{3} \\
N_{4}
\end{array}\right\} \cdot E J_{d} \cdot \frac{\partial^{4}}{\partial x^{4}}\left[\begin{array}{llll}
N_{1} & N_{2} & N_{3} & N_{4}
\end{array}\right]\left\{\begin{array}{c}
w_{1} \\
\varphi_{1} \\
w_{2} \\
\varphi_{2}
\end{array}\right\} \cdot d x=K_{w w} \cdot\left\{\begin{array}{c}
w_{1} \\
\varphi_{1} \\
w_{2} \\
\varphi_{2}
\end{array}\right\} \\
& \int_{0}^{L}\left\{\begin{array}{l}
N_{1} \\
N_{2} \\
N_{3} \\
N_{4}
\end{array}\right\} \theta \cdot E J_{d} \cdot \frac{\partial^{5}}{\partial x^{4} \partial t}\left[\begin{array}{llll}
N_{1} & N_{2} & N_{3} & N_{4}
\end{array}\right]\left\{\begin{array}{c}
w_{1} \\
\varphi_{1} \\
w_{2} \\
\varphi_{2}
\end{array}\right\} \cdot d x=\theta \cdot K_{w w} \cdot \frac{\partial}{\partial t}\left\{\begin{array}{l}
w_{1} \\
\varphi_{1} \\
w_{2} \\
\varphi_{2}
\end{array}\right\}
\end{aligned}
$$


in which:

$$
\begin{gathered}
K_{w w}=\frac{E J_{d}}{L^{3}}\left[\begin{array}{llll}
12 & 6 L & -12 & 6 L \\
6 L & 4 L^{2} & -6 L & 2 L^{2} \\
-12 & -6 L & 12 & -6 L \\
6 L & 2 L^{2} & -6 L & 4 L^{2}
\end{array}\right] ; \\
\int_{0}^{L}\left\{\begin{array}{l}
N_{1} \\
N_{2} \\
N_{3} \\
N_{4}
\end{array}\right\} \cdot \rho F_{d} \cdot \frac{\partial^{2}}{\partial t^{2}}\left[\begin{array}{llll}
N_{1} & N_{2} & N_{3} & N_{4}
\end{array}\right]\left\{\begin{array}{l}
w_{1} \\
\varphi_{1} \\
w_{2} \\
\varphi_{2}
\end{array}\right\} \cdot d x=M_{w w} \frac{\partial^{2}}{\partial t^{2}}\left\{\begin{array}{l}
w_{1} \\
\varphi_{1} \\
w_{2} \\
\varphi_{2}
\end{array}\right\} ; \\
\int_{0}^{L}\left\{\begin{array}{c}
N_{1} \\
N_{2} \\
N_{3} \\
N_{4}
\end{array}\right\} \cdot \beta \cdot \frac{\partial}{\partial t}\left[\begin{array}{llll}
N_{1} & N_{2} & N_{3} & N_{4}
\end{array}\right]\left\{\begin{array}{l}
w_{1} \\
\varphi_{1} \\
w_{2} \\
\varphi_{2}
\end{array}\right\} \cdot d x=\beta \cdot \frac{M_{w w}}{\rho F_{d}} \frac{\partial}{\partial t}\left\{\begin{array}{l}
w_{1} \\
\varphi_{1} \\
w_{2} \\
\varphi_{2}
\end{array}\right\} ;
\end{gathered}
$$

in which:

$$
\begin{aligned}
& M_{w w}=\frac{\rho F_{d} L}{420}\left[\begin{array}{llll}
156 & 22 L & 54 & -13 L \\
22 L & 4 L^{2} & 13 L & -3 L^{2} \\
54 & 13 L & 156 & -22 L \\
-13 L & -3 L^{2} & -22 L & 4 L^{2}
\end{array}\right] ; C_{w w}=\beta \cdot \frac{M_{w w}}{\rho F_{d}}+\theta \cdot K_{w w} ; \\
& \int_{0}^{L}\left\{\begin{array}{l}
N_{1} \\
N_{2} \\
N_{3} \\
N_{4}
\end{array}\right\} \cdot p(x, z, t) \cdot d x=\begin{array}{l}
{\left[-m_{21} . \ddot{z}_{21}+\left(\dot{z}_{11}-\dot{z}_{21}\right) d_{11}+\left(z_{11}-z_{21}\right) k_{11}\right] \cdot P_{1}+} \\
+\left[-m_{22} . \ddot{z}_{22}+\left(\dot{z}_{12}-\dot{z}_{22}\right) d_{12}+\left(z_{12}-z_{22}\right) k_{12}\right] \cdot P_{2}
\end{array}
\end{aligned}
$$

in which:

$$
P_{i}=\left\{\begin{array}{l}
p_{1 i} \\
p_{2 i} \\
p_{3 i} \\
p_{4 i}
\end{array}\right\}=\frac{\xi_{i}(t)}{L^{3}} \cdot\left\{\begin{array}{l}
\left(L+2 x_{i}\right)\left(L-x_{i}\right)^{2} \\
L \cdot x_{i}\left(L-x_{i}\right)^{2} \\
x_{i}^{2}\left(3 L-2 x_{i}\right) \\
-L \cdot x_{i}^{2}\left(L-x_{i}\right)
\end{array}\right\} .
$$

Combining the results (12) - (19) with (9) and rewriting in matrix form, we obtain:

$$
M_{e} \cdot \ddot{q}+C_{e} \cdot \dot{q}+K_{e} \cdot q=f_{e}
$$

$\ddot{q}, \dot{q}, q, f_{e}$ - the mixed acceleration vector, mixed velocity vector, mixed displacement vector, mixed forces vector, respectively:

$$
\{\ddot{q}\}=\left\{\begin{array}{c}
\ddot{w} \\
\ddot{z}_{1} \\
\ddot{z}_{2}
\end{array}\right\} ;\{\dot{q}\}=\left\{\begin{array}{c}
\dot{w} \\
\dot{z}_{1} \\
\dot{z}_{2}
\end{array}\right\} ;\{q\}=\left\{\begin{array}{c}
w \\
z_{1} \\
z_{2}
\end{array}\right\} ;\left\{f_{e}\right\}=\left\{\begin{array}{c}
f_{w} \\
f_{z_{1}} \\
f_{z_{2}}
\end{array}\right\}
$$

$M_{e}, C_{e}, K_{e}$ - the mixed quantity matrix, mixed damper matrix, mixed stiffness matrix, respectively:

$$
M_{e}=\left[\begin{array}{lll}
M_{w w} & 0 & M_{w z 2} \\
0 & M_{z 1 z 1} & 0 \\
0 & 0 & M_{z 2 z 2}
\end{array}\right] ; C_{e}=\left[\begin{array}{lll}
C_{w w} & C_{w z 1} & C_{w z 2} \\
0 & C_{z 1 z 1} & C_{z 1 z 2} \\
C_{z_{2} w} & C_{z 2 z 1} & C_{z 2 z 2}
\end{array}\right]
$$




$$
\begin{aligned}
& K_{e}=\left[\begin{array}{lll}
K_{w w} & K_{w z 1} & K_{w z 2} \\
0 & K_{z 1 z 1} & K_{z 1 z 2} \\
K_{z 2 w} & K_{z 2 z 1} & K_{z 2 z 2}
\end{array}\right] \\
& M_{w z_{2}}=P .\left[\begin{array}{ll}
m_{21} & 0 \\
0 & m_{22}
\end{array}\right] ; M_{z_{1} z_{1}}=P .\left[\begin{array}{ll}
m J & 0 \\
0 & m J
\end{array}\right] ; M_{z_{2} z_{2}}=\left[\begin{array}{ll}
m_{21} & 0 \\
0 & m_{22}
\end{array}\right] \text {; } \\
& P=\left[\begin{array}{ll}
P_{1} & P_{2}
\end{array}\right], P_{1}, P_{2} \text { are determined by (19); } \\
& C_{w z_{1}}=\left[\begin{array}{ll}
-d_{11} & 0 \\
0 & -d_{12}
\end{array}\right] ; C_{w z_{2}}=\left[\begin{array}{ll}
d_{11} & 0 \\
0 & d_{12}
\end{array}\right] ; \\
& C_{z_{1} z_{1}}=\left[\begin{array}{cc}
\left(a^{2} m+J\right) d_{11} & -(m b a-J) d_{12} \\
(m b a+J) d_{11} & \left(b^{2} m+J\right) d_{12}
\end{array}\right] ; C_{z_{1} z_{2}}=\left[\begin{array}{ll}
-\left(a^{2} m+J\right) d_{11} & (m b a-J) d_{12} \\
-(m b a+J) d_{11} & -\left(b^{2} m+J\right) d_{12}
\end{array}\right] ; \\
& C_{z_{2} w}^{T}=N_{a}\left[\begin{array}{ll}
-d_{21} & 0 \\
0 & -d_{22}
\end{array}\right] ; C_{z_{2} z_{1}}=\left[\begin{array}{ll}
-d_{11} & 0 \\
0 & -d_{12}
\end{array}\right] ; C_{z_{2} z_{2}}=\left[\begin{array}{ll}
d_{11}+d_{12} & 0 \\
0 & d_{12}+d_{22}
\end{array}\right] ; \\
& K_{w z_{1}}=\left[\begin{array}{ll}
-k_{11} & 0 \\
0 & -k_{12}
\end{array}\right] ; \mathrm{K}_{\mathrm{wz} 2}=\left[\begin{array}{ll}
k_{11} & 0 \\
0 & k_{12}
\end{array}\right] \text {; } \\
& K_{z_{1} z_{1}}=\left[\begin{array}{ll}
\left(a^{2} m+J\right) k_{11} & -(m b a-J) k_{12} \\
(m b a+J) k_{11} & \left(b^{2} m+J\right) k_{12}
\end{array}\right] \\
& K_{z_{1} z_{2}}=\left[\begin{array}{ll}
-\left(a^{2} m+J\right) k_{11} & (m b a-J) k_{12} \\
-(m b a+J) k_{11} & -\left(b^{2} m+J\right) k_{12}
\end{array}\right] \text {; } \\
& K_{z_{2} w}^{T}=N_{a}\left[\begin{array}{ll}
-k_{21} & 0 \\
0 & -k_{22}
\end{array}\right]+\dot{N}_{a} \cdot\left[\begin{array}{ll}
-d_{21} & 0 \\
0 & -d_{22}
\end{array}\right] \text {; } \\
& K_{z_{2} z_{1}}=\left[\begin{array}{ll}
-k_{11} & 0 \\
0 & -k_{12}
\end{array}\right] ; K_{z_{2} z_{2}}=\left[\begin{array}{ll}
k_{11}+k_{21} & 0 \\
0 & k_{12}+k_{22}
\end{array}\right] \text {; } \\
& N_{a}=\left[\begin{array}{ll}
N_{11} & N_{12} \\
N_{21} & N_{22} \\
N_{31} & N_{32} \\
N_{41} & N_{42}
\end{array}\right] ; \text { in which : }\left\{\begin{array}{l}
N_{1 i}=\frac{1}{L^{3}} \cdot\left(L^{3}-3 \cdot L \cdot x_{i}^{2}+2 \cdot x_{i}^{3}\right) ; \\
N_{2 i}=\frac{1}{L^{2}} \cdot\left(L^{2} \cdot x_{i}^{-} 2 \cdot L \cdot x_{i}^{2}+x_{i}^{3}\right) ; \\
N_{3 i}=\frac{1}{L^{3}} \cdot\left(3 \cdot L \cdot x_{i}^{2}-2 \cdot x_{i}^{3}\right) ; \\
N_{4 i}=\frac{1}{L^{2}} \cdot\left(x_{i}^{3}-L \cdot x_{i}^{2}\right) ;
\end{array}\right.
\end{aligned}
$$

$x_{i}$ is determined by the formula (1)

$$
f_{e}=\left\{\begin{array}{l}
0 \\
f_{z 1} \\
f_{z 2}
\end{array}\right\} ; \mathrm{f}_{\mathrm{z} 1}=\left\{\begin{array}{l}
J P-J m g-\left(m h+m_{21} h_{21}+m_{22} h_{22}\right) m a . \ddot{s} \\
J P-J m g-\left(m h+m_{21} h_{21}+m_{22} h_{22}\right) m b . \ddot{s}
\end{array}\right\} ; \quad f_{z 2}=\left\{\begin{array}{l}
-m_{21} g \\
-m_{22} g
\end{array}\right\} .
$$

\section{APPLICATION TO VIBRATION ANALYSIS OF BRIDGE STRUCTURE UNDER MOVING LOADS}

By digitizing bridge structures into basic elements, combining the research results above with finite element method and utilizing algorithms generally used in finite element method one can construct vibration differential equations for the whole system [19].

$$
M \cdot \ddot{Q}+C \cdot \dot{Q}+K \cdot Q=F
$$


$M, C, K$, which is the mixed quantity matrix, the mixed damper matrix, and the mixed stiffness matrix of the total system,

$\ddot{Q}, \dot{Q}, Q, F$, which is the mixed acceleration vector, the mixed velocity vector, the mixed displacement vector, and the mixed forces vector of the total system.

After inserting corresponding boundary conditions and initial conditions to (21), we can solve the set of equations (21) by the Runge- Kutta-Merson method on the computer.

An application in analyzing vibration of a three-span continuous steel girder bridge structure $(40 \mathrm{~m}+60 \mathrm{~m}+40 \mathrm{~m})$ can be condidered as follows:

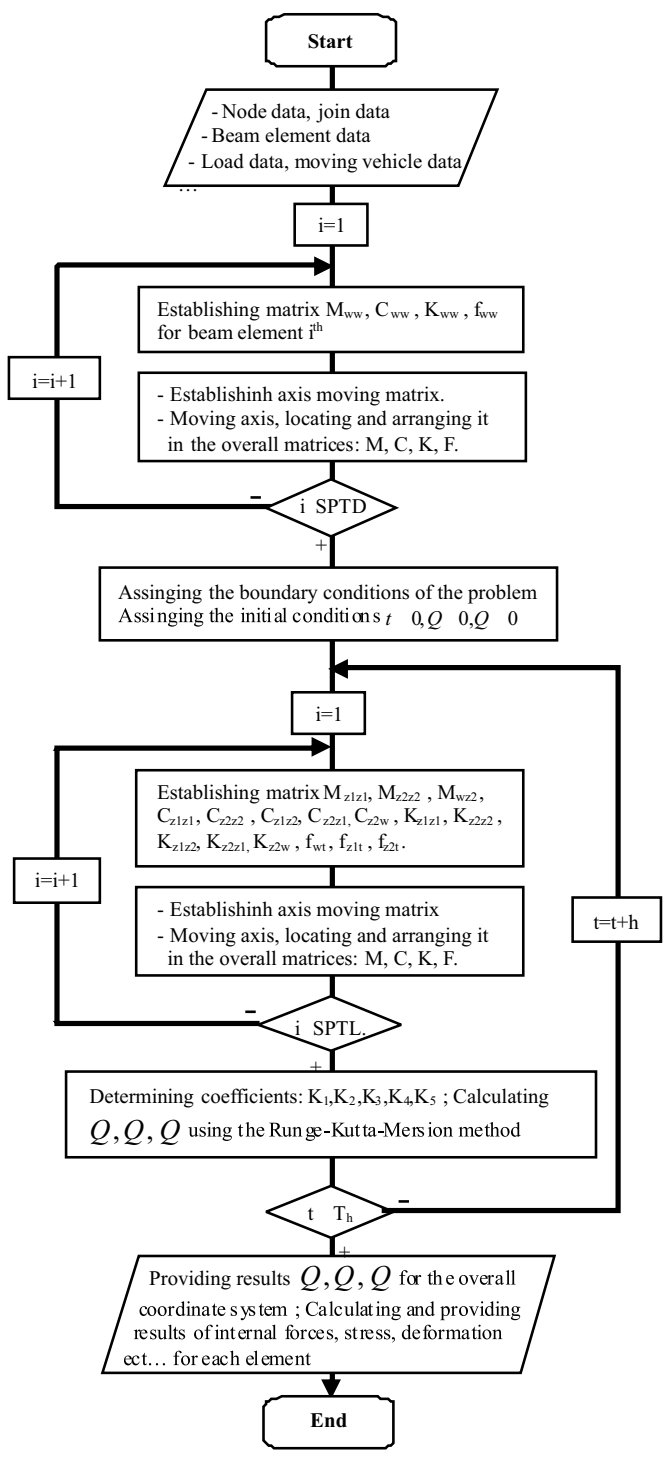

Fig. 3. The general algorithm in analyzing vibration of girder bridge structure 
Elastic module of steel $E=2.1 \times 10^{7} \mathrm{~T} / \mathrm{m}^{2}$, moment of inertia of area of steel girder $J_{d}=0.0261 \mathrm{~m}^{4}$, and mass of the beam element on a length unit $\rho F_{d}=1.237 \mathrm{~T} / \mathrm{m}$, friction factors $\theta=0.027 ; \beta=0.01 ; \tau=0.5$, acceleration of gravity $g=9.81 \mathrm{~m} / \mathrm{s}^{2}$, and SPTD $=14$; $\mathrm{SPTL}=14$, where SPTD is the number of beam elements of the whole structure, SPTL is the number of lane elements on the carriage-way, $T_{h}$ is the time period of analysis, $\mathrm{h}$ is time step (about $10^{-3} \mathrm{~s}$ ).

IFA-W50 trucks with following parameters: $m=9.838 \mathrm{~T},{ }_{m} 21=0.107 \mathrm{~T}, m_{22}=$ $0.055 \mathrm{~T}, P=0, a=1.035 \mathrm{~m}, b=2.415 \mathrm{~m}, h=1.5 \mathrm{~m}, h_{21}=0.5 \mathrm{~m}, h_{22}=0.5 \mathrm{~m}, k_{11}=200$ $\mathrm{T} / \mathrm{m}, k_{12}=30.2 \mathrm{~T} / \mathrm{m}, k_{21}=260 \mathrm{~T} / \mathrm{m}, k_{22}=120 \mathrm{~T} / \mathrm{m}, d_{11}=0.7344 \mathrm{Ts} / \mathrm{m}, d_{12}=0.3672$ $\mathrm{Ts} / \mathrm{m}, d_{21}=0.8 \mathrm{Ts} / \mathrm{m}, d_{22}=0.4 \mathrm{Ts} / \mathrm{m}$.

The general algorithm in analyzing vibration of girder bridge structure is shown in Fig. 3, where $K_{1}, K_{2}, K_{3}, K_{4}, K_{5}$ are coefficients calculated by using the Runge-KuttaMersion method.

Results of displacement calculation with velocity $3.6 \mathrm{~km} / \mathrm{h}$ are given in Figs. 4 - 7

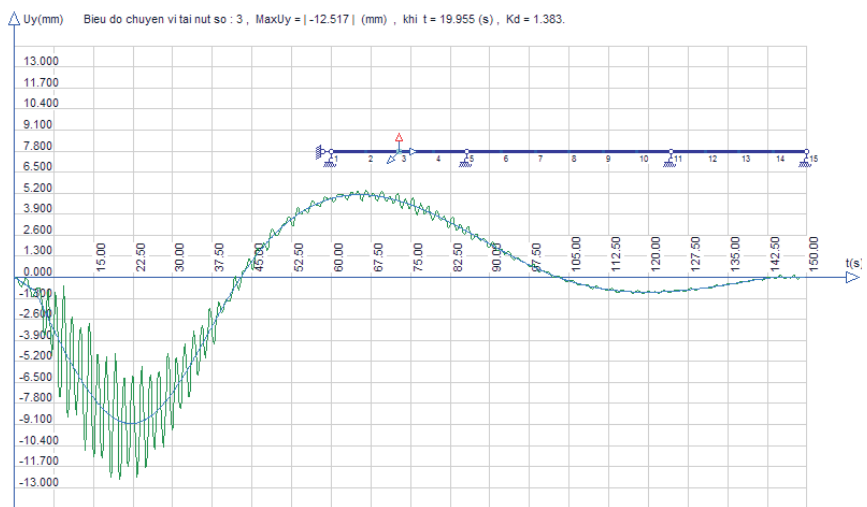

Fig. 4. At node 3, when not brake, $\mu=1.383$

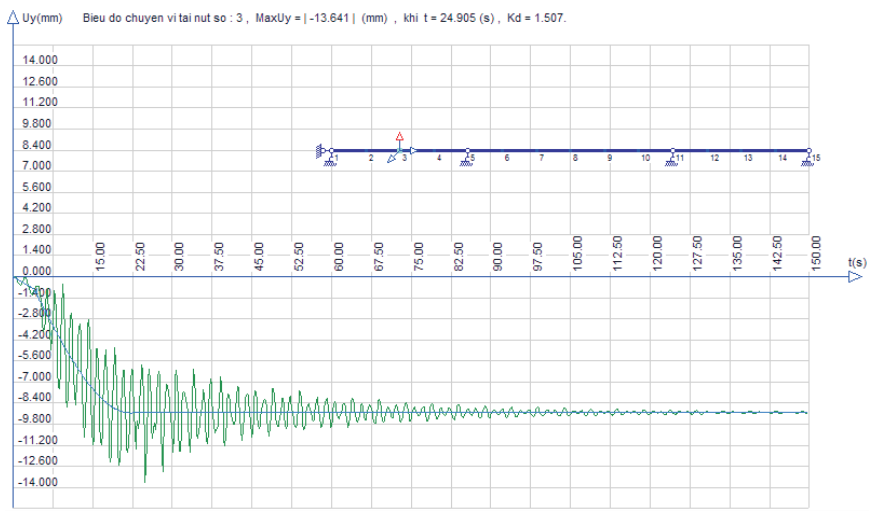

Fig. 5. At node 3, when not brake, $\mu=1.507$ 


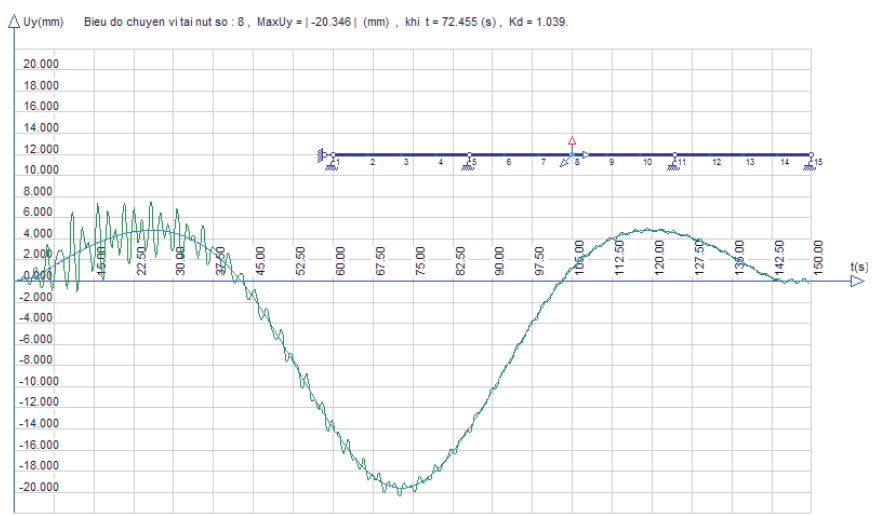

Fig. 6. At node 3, when not brake, $\mu=1.039$

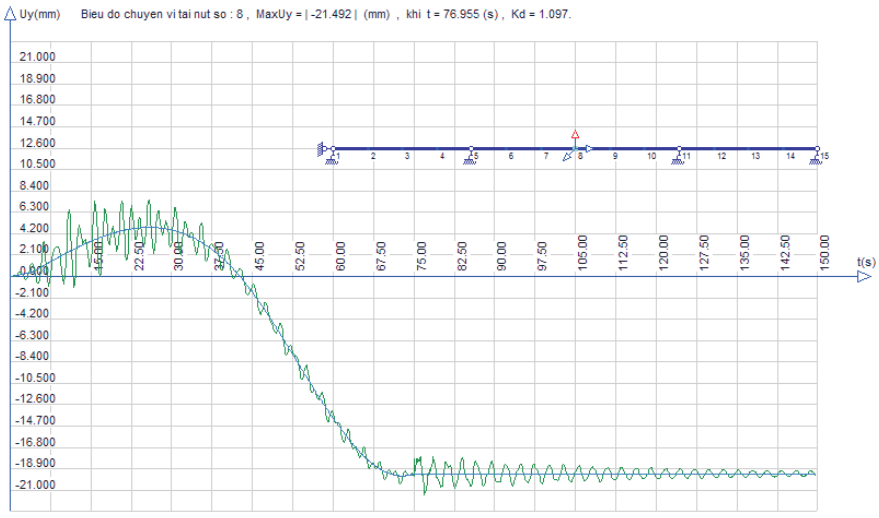

Fig. 7. At node 3, when not brake, $\mu=1.097$

Results of displacement calculation with velocity $10.8 \mathrm{~km} / \mathrm{h}$ are given in Figs. $8-11$

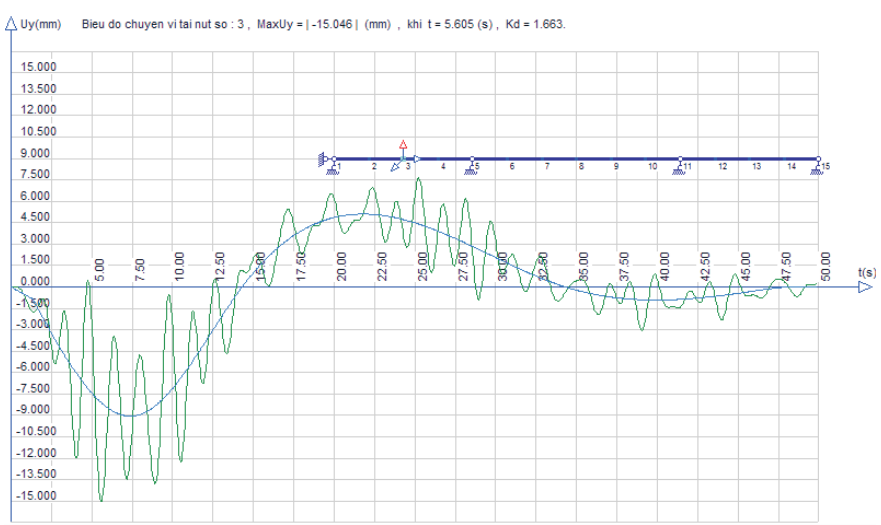

Fig. 8. At node 3, when not brake, $\mu=1.663$ 


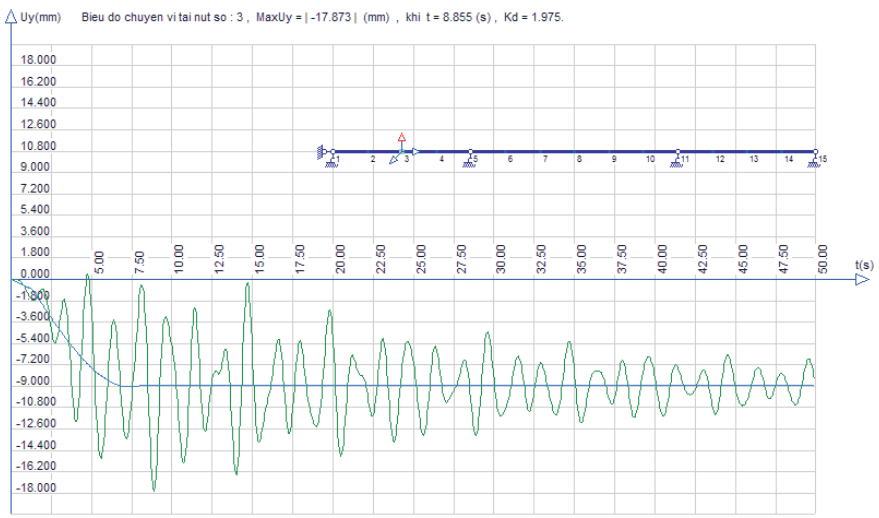

Fig. 9. At node 3, when not brake, $\mu=1.975$

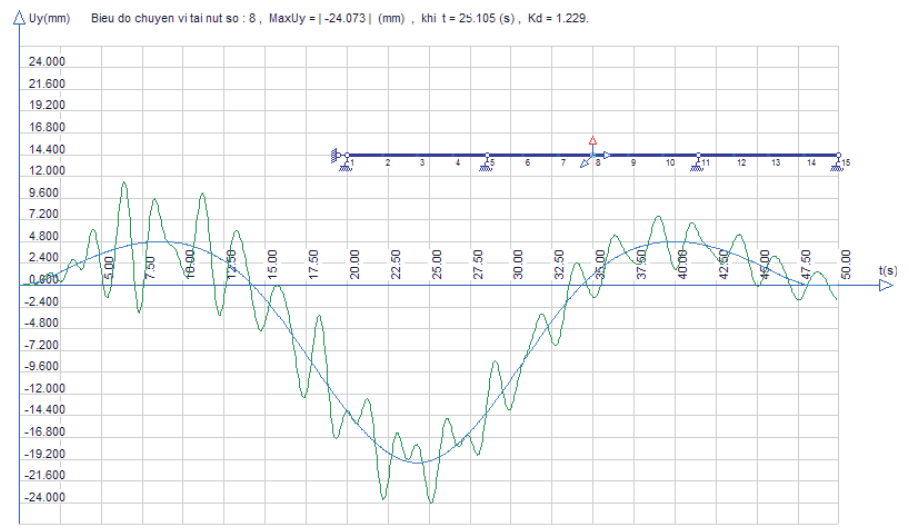

Fig. 10. At node 3, when not brake, $\mu=1.229$

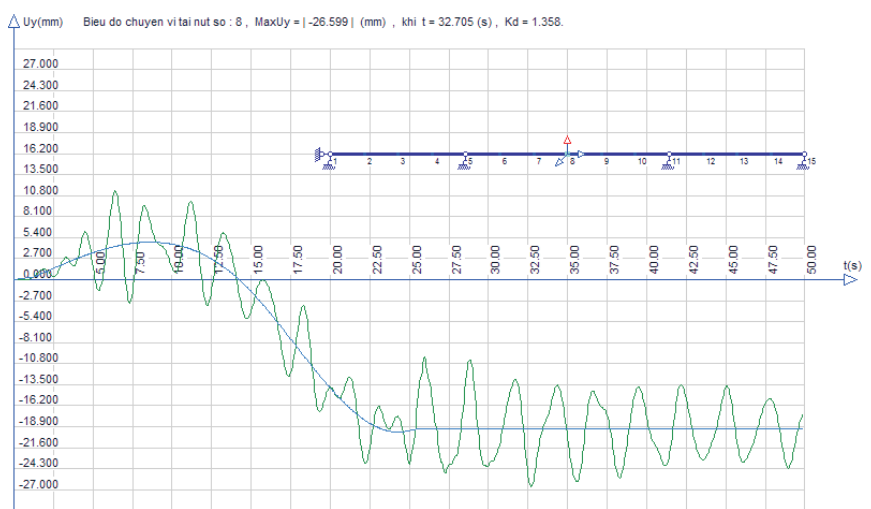

Fig. 11. At node 3, when not brake, $\mu=1.358$ 
In Figs 4 - 11, the follwings are denoted:

- the static displacement of girder,

- the dynamic displacement of girder,

$\mu \quad$ - the dynamic factor.

Calculation results show that the dynamic coefficient of deflection considering vehicle braking forces is bigger than that obtained without considering vehicle braking forces. Dynamic coefficient is significant and need to be considered in design calculations.

\section{CONCLUSIONS}

This paper introduces research results of building differential equations of bending vibrations of beam elements bearing moving loads considering vehicle braking forces and the way to build the combined stiffness matrices, combined mass matrices, mixed block matrices, equivalent force vector of beam elements bearing moving loads considering braking forces in accordance with a three-mass model. This research result is the basis for the study of bridge vibrations under the effect of moving loads of three - mass models considering the impact of vehicle braking force on the bridge.

\section{REFERENCES}

[1] Ministry of Transport, Standards 22TCN 272-05 for bridge design, Transportation Publisher, (2005) (in Vietnamese).

[2] AASHTO, Standard Specifications for Highway Bridges, 17th Edition, (2005).

[3] Hoang Ha, Research on bending vibrations of cable-stayed bridge structures on highways under the effect of moving load, Doctor thesis, Technology, Hanoi, (1999) (in Vietnamese).

[4] Do Xuan Tho, Calculation of bending vibrations of continuous beams under the effect of moving objects, Doctor thesis, Technology, Hanoi, (1996) (in Vietnamese).

[5] Nguyen Xuan Toan, Bending vibrations of beam elements under the effect of moving loads the model of one mass, Danang University Journal of Science and Technology, 2(14) (2006), 14-19 (in Vietnamese).

[6] Nguyen Xuan Toan, Analysis of cable-stayed bridge vibrations under the effect of moving loads, Doctor thesis, Technology, Hanoi, (2007) (in Vietnamese).

[7] Nguyen Xuan Toan, Research on building the software of analysis of dynamic interaction between cable-stayed bridges and fleets of moving loads of two mass model, Scientific and Technological Topic at the Ministerial Level, code SDH07-NCS-01, (2008) (in Vietnamese).

[8] Nguyen Xuan Toan, Nguyen Minh Hung, Algorithms and the program analysing horizontal vertical vibrations of beams and cable-stayed bridge towers under the effect of fleets of moving loads of two mass model, Report at the $8^{\text {th }}$ National Scientific Conference on Mechanics of Deformable Solids, Thai Nguyen, (2006), 834-843 (in Vietnamese).

[9] Nguyen Xuan Toan, Phan Ky Phung, The bending oscillation of beam elements in analysing vibrations in cable-stayed bridges under the effect of moving loads - two-quantity model, Journal of Transportation, 1,2 (2006), 105-107 (in Vietnamese).

[10] Nguyen Xuan Toan, Phan Ky Phung, Nonlinear oscillation of cable elements and their application to the analysis of cable-stayed bridges under the effect of moving load fleets, National Scientific Conference on Technical Mechanics and Automation, Hanoi, (2006), 281-290 (in Vietnamese). 
[11] Nguyen Xuan Toan, Phan Ky Phung, Nguyen Minh Hung, Analyzing the effects of speed and mass of a moving load on cable-stayed bridge oscillation, The $8^{\text {th }}$ National Mechanics Conference, Hanoi, (2007), 434-443 (in Vietnamese).

[12] Ta Huu Vinh, Hoang Xuan Luong, Anh Cuong Do, The impact of several factors on the interaction of bar- moving load systems, TTCT. The 7th National Scientific Conference on Mechanics of Deformable Solids, Publisher of Hanoi National University, (2004), 998-1008 (in Vietnamese).

[13] Nguyen Dong Anh, La Duc Viet, Do Anh Cuong, Vu Manh Lang, Pham Xuan Khang, Nguyen Ngoc Long, Analysis of structures with installation of energy dissipation devices, The $3^{\text {rd }}$ National Scientific Conference on the failure and damage of construction works, Construction Publisher, (2005), 29-38 (in Vietnamese).

[14] Curtis F. Gerald, Patrick O. Wheatley, Applied Numerical Analysis, Addison-Wesley, New York, (1999).

[15] N. X. Toan, P. K. Phung, Non-linear vibration of cable elements and applications in analysing the vibration of cable-stayed bridges under moving loads, International Conference on Modern Design, Construction and Maintenance of Structures, Hanoi Vietnam, 2 (2007), 37-43.

[16] Ray W. Clough and Joseph Penzien, Dynamics of structures, McGraw-Hill,Inc. Singapore, (1993).

[17] Yang Fuheng, Fonder Ghislain A., Dynamic response of cable-stayed bridges under moving loads, Journal of Engineering Mechanics, 124 (1998), 741-748.

[18] Zeman, M., Taheri, M. R., Khanna, A., Dynamic response of cable-stayed bridges to moving vehicles using the structure impedance method, Appl. Math. Modeling, 20 (1996), 877-889.

[19] Zienkiewicz O.C., Taylor R.L., The Finite Element Method, McGraw-Hill,Inc, New York, 1\&2 (1989). 